\title{
O uso da vibração como método auxiliar no treinamento de capacidades físicas: uma revisão da literatura
}

\author{
Camilla Zamfolini Hallal ${ }^{12}$ \\ Nise Ribeiro Marques ${ }^{12}$ \\ Mauro Gonçalves ${ }^{2} 3$ \\ ${ }^{1}$ Faculdade de Ciências e Tecnologia. UNESP - Univ Estadual Paulista, Campus de \\ Presidente Prudente, Presidente Prudente, SP, Brasil \\ ${ }^{2}$ Laboratório de Biomecânica. UNESP - Univ Estadual Paulista. Campus de Rio Claro, \\ Rio Claro, SP, Brasil \\ ${ }^{3}$ Instituto de Biociências. UNESP - Univ Estadual Paulista. Campus de Rio Claro, \\ Departamento de Educação Física, Rio Claro, SP, Brasil
}

\begin{abstract}
Resumo: Os efeitos da vibração sobre o corpo humano são documentados há muitos anos. Recentemente, o uso da vibração tem se destacado como método auxiliar no treinamento das capacidades físicas de indivíduos jovens, atletas e idosos. Estudos recentes indicam aumento no ganho de força, flexibilidade, resistência e potência de indivíduos treinados com equipamentos vibratórios, porém, os efeitos fisiológicos da vibração ainda são pouco entendidos, além de existir uma grande variedade dos protocolos de treinamento usados na literatura científica. A vibração apresenta-se como um importante estímulo ao sistema neuromuscular, entretanto, futuros estudos devem ser realizados como forma de se entender melhor os efeitos fisiológicos da vibração, bem como os efeitos de diferentes protocolos sobre o treinamento de capacidades físicas como força, flexibilidade, resistência e potência.
\end{abstract}

Palavras-chave: Vibração. Treinamento. Adaptação Neuromuscular.

\section{The use of vibration as an auxiliary method to train physical capabilities: a literature review}

\begin{abstract}
The effects of vibration on the human body have been documented for many years. Recently, the use of vibration is an auxiliary method of physical training in the young, elderly and athletes. Recent studies indicate an increase in gain of strength, flexibility, endurance and power of individuals trained with vibrating equipments. Despite the fairly widespread use, the physiological effects of vibration are still poorly understood, and there is great variability in the training protocols used in the literature. Future studies should be conducted as a way to better understand the physiological effects of vibration and also the affects of different protocols on physical training as strength, flexibility, endurance and power.
\end{abstract}

Key Words: Vibration. Training. Neuromuscular Adaptation.

\section{Introdução}

Vibração é um estímulo mecânico caracterizado por movimento oscilatório, no qual a intensidade varia de acordo com a freqüência, amplitude e magnitude do movimento gerado. Existem diversas formas de ondas provocadas pelo movimento oscilatório, como as ondas sinusoidais, transientes e estacionárias (Figura 1). A freqüência de vibração é mensurada em Hertz $(\mathrm{Hz})$ e representa o número de ciclos de movimento por segundo. A amplitude é caracterizada pelo deslocamento vertical da onda vibratória, sendo expressa em unidades de medidas de comprimento (milímetro, por exemplo). Além disso, a magnitude do movimento oscilatório é representada pela aceleração (g ou m. $\mathrm{s}^{-2}$ ) (CARDINALE; BOSCO, 2003; JORDAN et al., 2005).

Os efeitos prejudiciais da vibração sobre as estruturas biológicas têm sido bastante documentados na literatura. Altas freqüências e amplitudes de vibração associadas à exposição crônica por longos períodos de tempo causam danos importantes aos nervos periféricos, vasos sanguíneos e articulações. No entanto, recentemente, estudos com baixas freqüências e amplitudes de vibração têm mostrado efeitos positivos no treinamento e reabilitação das capacidades físicas de indivíduos de diversos níveis de condicionamento físico e faixa etária. Este tipo de vibração pode se aplicada diretamente sobre músculos e tendões, no corpo todo por meio de plataformas vibratórias e 
também por meio de hastes vibratórias (MATTHEW et al., 2005; SHINOHARA, 2005).

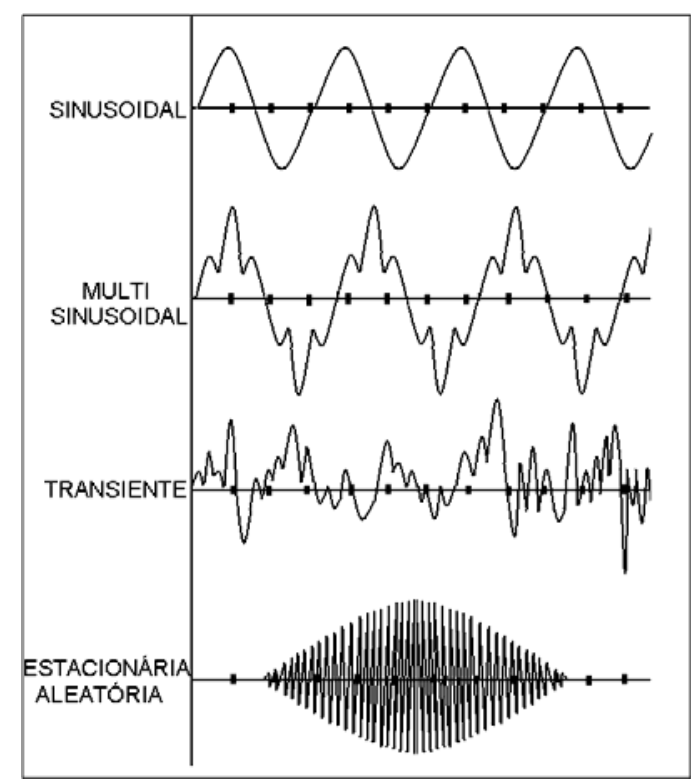

Figura 1. Exemplo de ondas com diferentes tipos de movimento oscilatório.

As plataformas vibratórias são o tipo de vibração mais usado atualmente e caracterizamse por produzir ondas sinusoidais com freqüências entre 15 e $60 \mathrm{~Hz}$ e deslocamento vertical ou horizontal variando de 1 a $10 \mathrm{~mm}$. As plataformas vibratórias promovem a vibração do corpo todo e devido a sua versatilidade proporcionam o acesso a inúmeros protocolos de treinamento com diversas combinações de freqüência, amplitude, tempo de exposição, entre outros. Estudos recentes mostraram os efeitos da vibração do corpo todo no treinamento de força, potência, flexibilidade e equilíbrio (TORVINEN et al., 2002; DELECLUSE et al., 2003; VERSCHUEREN et al., 2004; CORMIE, et al., 2006; ROELANTS et al., 2006; BOGAERTS et al., 2007; REES et al., 2008).

O treino com haste vibratória distingue-se dos demais treinos com vibração pela menor freqüência alcançada, cerca de $4,5 \mathrm{~Hz}$, e pelo comportamento passivo da haste, cuja vibração não é produzida por força externa, mas pela contração muscular. Estudos mostraram efeitos positivos do treinamento de indivíduos jovens, com hastes vibratórias, na força muscular, resistência e equilíbrio (SUGIMOTO, BLANPIED, 2006; ANDERS et al., 2007; BUTEAU et al., 2007; LISTER et al., 2007; MORESIDE, et al., 2007).

Apesar do uso bastante difundido no treinamento e reabilitação, os efeitos da vibração no corpo humano ainda são pouco entendidos, além de existir uma grande variabilidade nos protocolos de treinamento usados na literatura científica. Assim, no sentido de explorar a atuação da vibração no tecido músculoesquelético, o presente estudo teve por objetivo analisar, por meio de uma revisão de literatura, os efeitos da vibração como método auxiliar no treinamento de capacidades físicas.

\section{Efeitos metabólicos da vibração}

Atualmente, muitos estudos têm abordado os efeitos da vibração no sistema músculoesquelético, porém, pouco se sabe sobre a influência deste estímulo mecânico no metabolismo corporal (RITTWEGER et al., 2002; VERSCHUEREN et al., 2004; BAUM et al., 2007).

As contrações musculares rítmicas causadas pela vibração podem alterar alguns parâmetros cardiovasculares (KERSCHAN et al., 2001). Estudos revelam que estas alterações são mínimas e que após um curto período de tempo, cerca de 15 minutos, a pressão arterial e a freqüência cardíaca retornam ao normal, não provocando nenhum tipo de stress cardiovascular (RITTWEGER et al., 2000; HAZELL et al., 2008). Lohman et al. (2007) analisou os efeitos da vibração do corpo todo sobre o fluxo sanguíneo da pele em exercícios isométricos leves de curta duração e concluiu que o aumento do fluxo sanguíneo decorrente do estímulo vibratório não foi significativo.

O sistema neuroendócrino tem um importante papel na determinação da resposta individual ao exercício. Em particular, mudanças na concentração sanguínea de testosterona e cortisol têm relevância no efeito dos exercícios sobre as adaptações ósseas e musculares (KRAEMER; RATAMESS, 2005). Em estudo realizado por Bosco et al. (2000), foi constatado aumento na concentração plasmática de testosterona e diminuição de cortisol em indivíduos jovens submetidos vibração aguda do corpo todo. Entretanto, Erskine et al. (2007) após avaliar a concentração salivar de testosterona e cortisol após exercícios isométricos de semiagachamento com vibração do corpo todo, observaram que o estímulo vibratório não foi estressante para o sistema neuroendócrino, pois não houve mudança significativa na concentração destes hormônios.

Deste modo, evidencia-se que o estímulo mecânico da vibração, apesar de alterar minimamente alguns parâmetros metabólicos, 
não provoca nenhum tipo de stress aos sistemas fisiológicos. No entanto, indivíduos com qualquer tipo de comprometimento metabólico, devem ser minuciosamente avaliados e acompanhados durante o treinamento com equipamentos vibratórios.

\section{Reflexo tônico de vibração}

O controle do movimento humano depende da atividade das unidades motoras, determinada pela integração entre os estímulos neurais excitatórios e inibitórios para o neurônio motor a (CARDINALE; BOSCO, 2003; JORDAN et al., 2005; SHINOHARA, 2005). O comando motor excitatório do córtex, as fibras inibitórias aferentes do tipo lb dos órgãos tendinosos de Golgi (OTG) e as fibras excitatórias aferentes do tipo la dos fusos musculares, são os principais estímulos neurais para o neurônio motor a (SHINOHARA, 2005).

Os fusos musculares são sensíveis a mudanças no comprimento das fibras musculares. O estímulo vibratório induz repetidas alterações no comprimento das fibras musculares, o que aumenta a taxa de disparo das fibras aferentes do tipo la com conseqüente excitação dos neurônios motores $\alpha$. Deste modo, ocorre a contração de unidades motoras homônimas, causando o fenômeno conhecido como reflexo tônico de vibração (JORDAN et al., 2005; SHINOHARA, 2005). A Figura 2 ilustra, simplificadamente, as conexões neurais que podem ser influenciadas pela vibração.

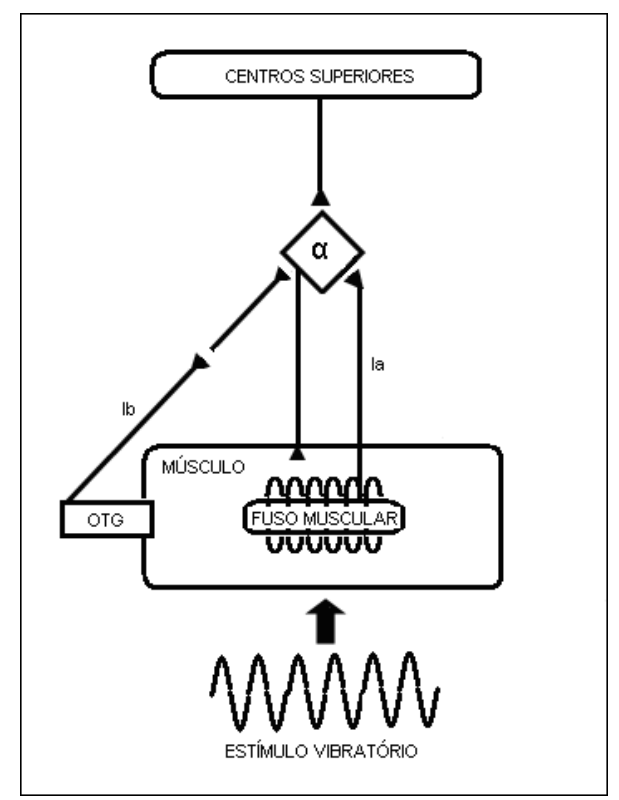

Figura 2. Conexões neurais influenciadas pela vibração.

A resposta tônica reflexa da vibração está relacionada a muitos fatores como a freqüência de vibração, o nível de pré-contração muscular, a posição do segmento, além do tipo e do local de aplicação do estímulo vibratório (ROHMERT et al., 1989; MARTIN; PARK, 1997; PARK; MARTIN, 1998). Assim, apesar dos efeitos da vibração sobre o músculo esquelético serem bastante documentados na literatura, o reflexo tônico de vibração não é necessariamente observado nos treinos com equipamentos vibratórios quando os fatores descritos acima não são adequadamente controlados.

\section{Efeitos da vibração sobre o sistema músculo esquelético}

Recentemente, a vibração como método auxiliar no treinamento das capacidades físicas de indivíduos jovens, atletas e idosos, tem despertado grande interesse na comunidade científica. A eletromiografia de superfície (EMG), por meio do monitoramento dos valores de root mean square (RMS) é uma técnica muito utilizada para evidenciar a resposta muscular decorrente da intervenção com equipamentos vibratórios. Moras et al. (2006) após análise eletromiográfica dos músculos do quadríceps expostos a diferentes cargas vibratórias, concluiu que os valores de RMS para o músculo vasto medial foi significativamente maior nas condições com vibração do que sem a presença deste estímulo. Em estudo realizado por Cardinale e Lim (2003), foi demonstrado um aumento significativo da atividade eletromiográfica do músculo vasto lateral durante a exposição à vibração em posição de semi-agachamento, em relação a mesma condição sem vibração.

A vibração do corpo todo, por meio de plataformas vibratórias, tem sido empregada no treinamento de força e potência. Cormie et al. (2006) e David et al. (2008) analisaram por meio de exercícios isométricos e saltos, os efeitos agudos da vibração do corpo todo na atividade muscular, força, e potência dos músculos da perna. Ambos concluíram que houve um aumento significativo na força, potência e atividade dos músculos analisados nas condições de exercícios executados com a plataforma vibratória. Em estudos realizados por Rees et al. (2007) foi analisado o impacto do treinamento com plataforma vibratória sobre o desempenho muscular de idosos, e conclui-se que o treinamento com vibração não obteve diferença significativa no desempenho muscular da população analisada, quando comparado com o treinamento sem vibração.

Os efeitos da vibração também têm sido investigados em estudos que envolvem a 
aplicação da vibração durante exercícios para ganho de mobilidade (BAUTMANS et al., 2005). Sands et al. (2006) observou a influência da vibração sobre 0 alongamento estático de voluntários ginastas e concluiu que ocorreram efeitos positivos, a curto e longo prazo, no ganho de amplitude de movimento dos voluntários. Fagnani et al. (2006) implementou um protocolo de 8 semanas de treinamento com plataforma vibratória para atletas do sexo feminino e concluiu que a vibração foi eficaz no ganho de flexibilidade desta população.

Além dos equipamentos de vibração local e do corpo todo, as hastes vibratórias também vêm sendo exploradas pela comunidade científica devido aos seus efeitos na ativação muscular. As rápidas contrações musculares concêntricas e excêntricas advindas da movimentação da haste geram co-contração dos grupos musculares envolvidos, o que aumenta o controle central e periférico da posição articular. Lister et al. (2007) identificaram por meio da EMG, que os músculos supraespinhal, trapézio fibras superiores e trapézio fibras inferiores possuem maior atividade durante exercícios executados com o uso de haste vibratória do que com faixa elástica ou cargas livres.

\section{Variabilidade nos protocolos de vibração}

As características dos protocolos de treinamento e intervenção com vibração podem variar em diversos aspectos como: as características da vibração (amplitude e freqüência das ondas), o tipo de equipamento utilizado (plataformas, hastes vibratórias e vibradores locais), o tempo de exposição à vibração, o local de aplicação da vibração, o tempo e a freqüência do treinamento, entre outros (JORDAN et al., 2005).

Em estudos realizados por Kitazani e Griffin (1998) foi mostrado que o grau de contração muscular e a posição do corpo durante a exposição à vibração podem afetar a resposta biológica. Martin e Park (1997) apontaram que o desencadeamento do reflexo tônico de vibração está diretamente relacionado com a freqüência de vibração imposta ao tecido muscular. Em estudo realizado por Bongiovanni et al. (1990) sugeriu-se que a duração da exposição à vibração pode afetar grandemente a função muscular. Deste modo, as evidências científicas apontam variações importantes que devem ser consideradas e controladas para uma maior viabilidade da intervenção com vibração.

A Tabela 1 mostra a diferença entre os protocolos de vibração usados em estudos anteriores.

Tabela 1. Comparação entre os diferentes protocolos de vibração utilizados em estudos anteriores

\begin{tabular}{|c|c|c|c|}
\hline Autor & Equipamento & Freqüência & Amplitude \\
\hline Santos et al., 2008 & Plataforma vibratória & $20 \mathrm{~Hz}$ & - \\
\hline Rees et al., 2008 & Plataforma vibratória & $26 \mathrm{~Hz}$ & $5 ; 6 ; 7 ; 8 \mathrm{~mm}$ \\
\hline Cormie et al., 2006 & Plataforma vibratória & $30 \mathrm{~Hz}$ & $2,5 \mathrm{~mm}$ \\
\hline Roelants et al., 2006 & Plataforma vibratória & $35 \mathrm{~Hz}$ & $2,5 \mathrm{~mm}$ \\
\hline David et al., 2008 & Plataforma vibratória & $30 ; 35 ; 40 ; 50 \mathrm{~Hz}$ & $2-6 \mathrm{~mm}$ \\
\hline$\overline{\text { Delecluse et al., } 2005}$ & Plataforma vibratória & $35 ; 40 \mathrm{~Hz}$ & 2,$5 ; 4 \mathrm{~mm}$ \\
\hline$\overline{\text { Roelants et al., 2004a }}$ & Plataforma vibratória & $35 ; 45 \mathrm{~Hz}$ & 2,$5 ; 5 \mathrm{~mm}$ \\
\hline$\overline{\text { Roelants et al., } 2004 \mathrm{~b}}$ & Plataforma vibratória & $35 ; 45 \mathrm{~Hz}$ & 2,$5 ; 5 \mathrm{~mm}$ \\
\hline$\overline{\text { Delecluse et al., } 2003}$ & Plataforma vibratória & $35 ; 40 \mathrm{~Hz}$ & 1,$7 ; 2,5 \mathrm{~mm}$ \\
\hline Rees et al., 2007 & Plataforma vibratória & $26 \mathrm{~Hz}$ & $5 ; 6 ; 7 ; 8 \mathrm{~mm}$ \\
\hline$\overline{\text { Fagnani et al., } 2006}$ & Plataforma vibratória & $35 \mathrm{~Hz}$ & $4 \mathrm{~mm}$ \\
\hline Yoshitake et al., 2004 & Vibrador local & $100 \mathrm{~Hz}$ & $0,75 \mathrm{~mm}$ \\
\hline Ushiyama et al.; 2005 & Vibrador local & $80-100 \mathrm{~Hz}$ & $1,5 \mathrm{~mm}$ \\
\hline Ivanenko et al., 2000 & Vibrador local & $80 \mathrm{~Hz}$ & $1,5 \mathrm{~mm}$ \\
\hline Bongiovani et al., 1990 & Vibrador local & $150 \mathrm{~Hz}$ & $1,5 \mathrm{~mm}$ \\
\hline Griffin et al., 2001 & Vibrador local & $110 \mathrm{~Hz}$ & $3 \mathrm{~mm}$ \\
\hline Hultborn et al., 1987 & Vibrador local & $200 \mathrm{~Hz}$ & - \\
\hline Bove et al., 2003 & Vibradot local & $90 \mathrm{~Hz}$ & $0,9 \mathrm{~mm}$ \\
\hline Sands et al., 2006 & Vibração local & $30 \mathrm{~Hz}$ & $2 \mathrm{~mm}$ \\
\hline Moreside et al., 2007 & Haste vibratória & $4,5 \mathrm{~Hz}$ & - \\
\hline Anders et al., 2007 & Haste vibratória & $3 ; 3,5 ; 4,5 \mathrm{~Hz}$ & - \\
\hline
\end{tabular}




\section{Conclusão}

A literatura aponta grande divergência entre os efeitos agudos e crônicos da vibração no corpo humano, o que deve-se principalmente a grande variedade dos protocolos experimentais. Deste modo, as atualizações da literatura devem ser consideradas para a implementação adequada dos protocolos de treinamento com vibração e para o controle de parâmetros como freqüência de amplitude das ondas, tipo de equipamento adequado, tempo e local de exposição à vibração e freqüência de treinamento; os quais podem interferir diretamente nos efeitos fisiológicos proporcionados por este estímulo mecânico.

A presente revisão nos permite concluir que a vibração pode ser um importante estímulo para o sistema neuromuscular, atuando como instrumento auxiliar no treinamento e reabilitação de capacidades físicas, como força, flexibilidade, resistência e potência. No entanto, futuros estudos devem ser realizados acerca dos efeitos agudos e crônicos do treinamento com vibração, bem como o efeito de diferentes parâmetros vibratórios no desempenho músculo-esquelético.

\section{Referências}

ANDERS, C; WENZEL, B; SCHOLLE, H. C. Cyclic upper body perturbations caused by a flexive pole: influence of oscillation frequency and direction on trunk muscle co-ordination. Journal of Back and Musculoskeletal Rehabilitation, $v$. 20, p. 167-175, 2007.

BAUM, K; VOTTELER, T; SCHIAB, J. Efficiency of vibration exercise for glycemic control in type 2 diabetes patients. International Journal of Medical Sciences, v. 4, p. 159-163, 2007.

BAUTMANS, I; HEES, E. V; LEMPER, J. C; METS, T. The feasibility of whole body vibration in institutionalized elderly person and its influence on muscle performance, balance and mobility: a randomized controlled trial. BCM Geriatrics, v. 5, p. 1-8, 2005.

BOGAERTS, A; VERSCHUEREN, S; DELECLUSE, C; CLAESSENS, A. L; BOONEN, $S$. Effects of whole body vibration training of postural control in older individuals: a 1 yar randomized controlles trial. Gait \& Posture, v. 26, p. 309-316, 2007.

BONGIOVANNI, L. G; HAGBARTH, K. E; STJERNBERG, L. Prolonged muscle vibration reducing motos output in maximal voluntary contractions in man. Journal of Physiology, $v$. 423, p. 15-26, 1990.

BOSCO, C; JACOVELLI, M; TSARPELA, O; CARDINALE, M; BONIFAZI, M; TIHANYI, J; VIRU, M; LORENZO, A; VIRU, A. Hormonal responses to whole body vibration in man.

European Journal of Applied Physiology, v. 81, p. $449-454,2000$.

BOVE, M; NARDONE, A; SCHIEPPATI, M. Effects of leg muscle tendon vibration on group la and group II reflex responses to stance perturbation in humans. Journal of Physiology, v. 550, p. 617-630, 2003.

BUTEAU, J. L; ERIKSRUD, O; HASSON, S. M. Rehabilitation of a glenohumeral instability utilizing the body blade. Physioterapy Theory end Pratice, v. 23, p. 333-349, 2007.

CARDINALE, M; BOSCO, C. The use of vibration as an exercise intervention. Exercise and Sport Science Reviews, v. 31, p. 3-7, 2003.

CARDINALE, M; LIM, J. Electromiography activity of vastus lateralis muscle during whole body vibrations of different frequencies. European Journal of Applied Physiology, v. 17, p. 621624, 2003.

CORMIE, P; DEANE, R. S; TRIPLETT, T; MECBRIDE, J. Acute effects of whole body vibration on muscles activity, strength, and power. Journal of Strenght and Conditioning Research, v. 20, p. 257-261, 2006.

DAVID, M; BAZETT, J; HOLMES, W; DUGAN, E.L. Comparing the effects of various whole body vibration accelerations on counter movement jump performance. Journal of Sports Science and Medicine, v. 7, p. 144-150, 2008.

DELECLUSE, C; ROELANTS, M; DIELS, R; KONINCKX, E; VERSCHUEREN, S. Effects of wholw body vibration training os muscles strenght and sprint performance in sprint trained athletes. International Journal of Sports Medicine, v. 26, p. 662-668, 2005.

DELECLUSE, C; ROELANTS, M; VERSCHUEREN, S. Strenght increase after whole body vibration compared with resistance training. Medicine \& Science in Sports \& Exercise, v. 35, 0. 1033-1041, 2003.

ERSKINE, J; SMILLIE, I; LEIPER, J; BALL, D; CARDINALE, M. Neuromuscular and hormonal responses to a single session of whole body vibration exercise in healthy young men. Clinical Physiology and Functional Imaging, v. 27, p. 242-248, 2007. 
FAGNANI, F; GIOMBINI, A; CESARE, A; PIGOZZI, F; SALVO, V. The effects of a whole body vibration program on muscles performance and flexibility in female athletes. American Journal of Physical Medicine \& Rehabilitation, v. 85, p. $956-962,2006$.

GRIFFIN, L; GARLAND, S. J; IVANOVA, T; GOSSEN, E. R. Muscle vibration sustains motor unit firing rate during submaximal isometric fatigue in humans. Journal of Physiology, v. 585, p. 929-936, 2001.

HAZELL, T. J; GRAEME, W. R; THOMAS, J. R; LEMON, O. W. R. Vertical whole body vibration does not increase cardiovascular stress to static semi-squat exercise. Journal os Applied Physiology, v. 104, p. 903-908, 2008.

HULTBORN, H; MEUNIER, S; DESEILLIGNY, P. E; SHINDO, M. Changes in pre-synaptic of la fibres et the onset of voluntary contraction in man. Journal of Physiology, v. 389, p. 757-772, 1987.

IVANKENKO, Y. P; GRASSO, R; LACQUANITI, $F$. Influence of leg muscle vibration on human walking. Journal of Neurophysiology, v. 87, p. 1737-1747, 2000.

JORDAN, M. J; STHEPHEN, R. N; DAVID, J. S; HERZOG, W. Vibration training: an overview of the area, training consequences, and future considerations. Journal of Strenght and Conditional Research, v. 19, p. 459-466, 2005.

KERSCHAN, S. K; GRAMPP, S.H. C. Whole body vibration exercise leads to alterations in muscles blood volume. Clinical Physiology, v. 21, p. 377382, 2001.

KITAZANI, S; GRIFFIN, M. J. Ressonance behavior of the seated human body and effects of posture. Journal of Biomechanics, v. 31, p. 143149, 1998.

KRAEMER, W. J; RATAMESS, N. A. Hormonal responses and adaptations to resistance exercise and training. Sports Medicine, v. 35, p. 339-361, 2005.

LISTER, J. L; ROSSI, G. D; STOUTENBERG, M; ADAMS, J. B; TOBKIN, S; SIGNORILE, J. Scapular stabilizer activity during Bodyblade, cuff weights, and Thera-band use. Journal of Sports and Rehabilitation, v. 16, p. 50-57, 2007.

LOHMAN, E. B; PETROFSKY, J. S; HINDS, C. M; SCHWAB, H. B; THORPE, D. The effect of whole body vibration on lowe extremity skin blood flow in normal subjects. Medical Science Monitor, v. 13, p. 71-76, 2007.
MARTIN, B. J; PARK, H. S. Analyses of the tonic vibration reflex: influence of vibration variables on motor unit synchronization and fatigue. European Journal of Applied Physiology, v. 75, p. 504511, 1997.

MATTHEW, J. J; STEPHEN, R; DAVID, J. S; HERZOG, W. Vibration training: an overview of the area, training consequences, and future considerations. Journal of Strengh and Conditioning Research, v. 19, p. 459-466, 2005.

MORAS, G; TOUS, J; MUNOZ, C. J; PADULLES, J. M; VALLEJO, L. Electromyographic response during whole-body vibrations of different frequencies with progressive external loads. Revista Digital Buenos Aires, v. 93, 2006.

MORESIDE, I. M; GARCIA, F. V; MACGLIL, S. M. Trunk muscle activation patterns, lumbar compressive forces, and spine stability when using the Bodyblade. Physical Therapy, v. 87, p. 153-164, 2007.

PARK, H. S; MARTIN, B. J. Contribution of the tonic vibration reflex to muscle stress and muscle fatigue. Scandinavian Journal of Work Enviroment \& Health, v. 19, p. 35-42, 1998.

REES, S. S; MURPHY, A. J; WATSFORD, M. L. Effects of whole body vibration exercise on lowerextremity muscle strength and power in an older population: a randomized clinical trial. Physical Therapy, v. 88, p. 462-470, 2008.

REES, S. S; MURPHY, A. J; WATSFORD, M. L. Effects of vibration exercise on muscle performance and mobility in an older population. Journal of Aging and Physical Activity, v. 15, p. 367-381, 2007.

RITTWEGER, J; EHRIG, J; JUST, K. Oxygen uptake in whole-body vibration exercise: influence of vibration frequency, amplitude, and external load. International Journal of Sports Medicine, v. 23, p. 428-432, 2002.

RITTWEGER, G. B; FELSENBERG, D. Acute physiological effects of exhaustive wholebody vibration exercise in man. Clinical Physiology, v. 20, p. 134-142, 2000.

ROELANTS, M; DELECLUSE, C; GORIS, M; VERSCHUEREN, S. Effects of 24 weeks of whole body vibration training on body composition and muscles strnght in untrained females.

International Journal of Sports Medicine, v. 25, p. 1-5, 2004a.

ROELANTS, M; DELECLUSE, C; SABINE, M; VERSCHUEREN, S. Whole body vibration training increases knee extension strength and speed of movement in older women. Journal of 
American Geriatrics Society, v. 52, p. 901-908, 2004b.

ROELANTS, M; VERSCHUEREN, S; DELECLUSE, C; LEVIN, O; STIJNEN, V. Whole body vibration induced increased in leg muscle activity during different squat exercise. Journal of Strenght and Conditioning Reseach, v. 20, p. 124-129, 2006.

ROHMERT, W; WOS, H; NORLANDER, S; HELBIG, R. Effects of vibrations on arm and shoulder muscles in three body postures.

European Journal of Applied Physiology, v. 59, p. 243-248, 1989.

SANDS, W. A; MCNEAL, J. R; STONE, M. H; RUSSEAL, E. M; JEMNI, M. Flexibility enhancement with vibration: acute and long term. Medicine \& Science in Sports \& Exercise, v. 38, p. 720-725, 2006.

SANTOS, B. R; LARIVIERE, C; DELISLE, A; PLAMONDON, A; BOILEAU, P. E; IMBEAU, D. A. A laboratory study to quantify the biomechanical responses to whole body vibration: the influence on balance, relex response, muscular activity and fatigue. International Journal of Industrial Ergonomics, v. 38, p. 626-639, 2008.

SHINOHARA, M. Effects of prolonged vibration on motor unit activity and motor performance.

Medicine \& Science in Sports \& Exercise, v. 37, p. 2120-2125, 2005.

SUGIMOTO, D; BLANPIED, P. Flexible foil exercise and shoulder internal and external rotation strength. Journal of Athletic Training, v. 41 , p. 280-285, 2006.

TORVINEN, S; KANNUS, P; SIEVANEN, H; JARVINEN, T. A. H; PASANEN, M;

KONTULAINEM, S. Effect of four month vertical whole body vibration on performance an balance. Medicice \& Science in Sports \& Exercise, v. 39, p. 1532-1528, 2002.

USHIYAMA, J; MASANI, K; KOUZAKI, M; KANEHISA, H; FUKUNAGA, T. Difference in after effects following prolonged Achilles tendon vibration on muscles activity during maximal voluntary contraction among plantar flexor sinergists. Journal of Applied Physiology, v. 98, p. 1427-1433, 2005.

VERSCHUEREN, S. M. P; ROCLANTS, M; DELECLUSE, C; SWINNEN, S; VANDERSCHUEREN, D; BOONEN, S. Effect of 6 month whole body vibration training on hip density, muscles strength, and postural control in postmenopausal women: a randomized controlled pilot study. Journal of Bone an Mineral

Reseach, v. 19, p. 352-359, 2004.
YOSHITAKE, Y; SHINOHARA, M; KOUZAKI, M; FUKUNAGA, T. Flutuations in plantar flexion force are reduced after prolonged tendon vibration. Journal of Applied Physiology, v. 97, p. 20902097, 2004.

Endereço:

Mauro Gonçalves

Laboratório de Biomecânica, IB/Unesp

Av. 24-A, 1515 Bela Vista

Rio Claro SP Brasil

13506-900

Tel: (19) 3526.4345

e-mail: maurog@rc.unesp.br

Recebido em: 17 de dezembro de 2009.

Aceito em: 11 de março de 2010.

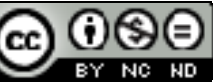

Motriz. Revista de Educação Física. UNESP, Rio Claro, SP, Brasil - elSSN: 1980-6574 - está licenciada sob Licença Creative Commons 